Article

\title{
The Modeling and Simulation of the Galvanic Coupling Intra-Body Communication via Handshake Channel
}

\author{
Maoyuan Li, Yong Song *, Wansong Li, Guangfa Wang, Tianpeng Bu, Yufei Zhao and Qun Hao \\ School of Optoelectronics, Beijing Institute of Technology, Beijing Key Laboratory for Precision Optoelectronic \\ Measurement Instrument and Technology, Beijing 100081, China; 2932183@bit.edu.cn (M.L.); \\ walsonlee1991@163.com (W.L.); zxcvbmn2009@126.com (G.W.); butianpeng@outlook.com (T.B.); \\ zhaoyufei@bit.edu.cn (Y.Z.); qhao@bit.edu.cn (Q.H.) \\ * Correspondence: yongsong@bit.edu.cn; Tel.: +86-10-6891-1760
}

Academic Editors: Giancarlo Fortino, Hassan Ghasemzadeh, Wenfeng Li, Yin Zhang and Luca Benini Received: 26 January 2017; Accepted: 10 April 2017; Published: 14 April 2017

\begin{abstract}
Intra-body communication (IBC) is a technology using the conductive properties of the body to transmit signal, and information interaction by handshake is regarded as one of the important applications of IBC. In this paper, a method for modeling the galvanic coupling intra-body communication via handshake channel is proposed, while the corresponding parameters are discussed. Meanwhile, the mathematical model of this kind of IBC is developed. Finally, the validity of the developed model has been verified by measurements. Moreover, its characteristics are discussed and compared with that of the IBC via single body channel. Our results indicate that the proposed method will lay a foundation for the theoretical analysis and application of the IBC via handshake channel.
\end{abstract}

Keywords: intra-body communication; galvanic coupling; transfer function; Information interaction

\section{Introduction}

Intra-body communication (IBC) is a technology using the conductive properties of the human body to transmit signals [1], thus providing a novel communication method among wearable sensors in body sensor networks (BSN) [2-4]. Compared with current short-distance wireless technologies, such as Bluetooth and Zigbee, IBC technology has the advantages of high reliability, high speed, low consumption, and security [5-7]. As a result, real-time biomedical monitoring can be achieved by surface-mounted and implantable biomedical devices using IBC technology [7]. The other important application of IBC technology is that two individuals can exchange data by shaking hands without complicated operations. In this application, when two individuals shake hands, one individual can automatically acquire the other's electric business cards through the handshake channel. Moreover, IBC technology also can be used for transferring multimedia data, creating human ad-hoc networks, and providing an assistance system for handicapped people [8,9].

In the literature, the scenario of IBC via handshake channel originated with Zimmerman [10]. Then, Shinagawa et al. designed a transceiver based on the electro-optic effect, and carried out an IBC experiment involving signal transmission via shaking hands [5]. Further to this, Hachisuka et al. measured signal transmission gains between two persons by shaking hands [11]. Shimamoto et al. measured the differences in received signal power attenuation between two individuals who touched, compared to two individuals who did not touch [8]. It should be noted that the above works were analyzed using the model of IBC via a single body channel, rather than the model of the IBC via handshake channel. Actually, the model of the IBC via handshake channel is very important for 
determining its characteristics, which will help to guarantee the safety of human bodies as well as the design of its application system [12-15]. However, it is generally not easy to develop a mathematical model galvanic coupling intra-body communication via handshake channel. Firstly, compared with IBC models via a single body channel, models of this kind of IBC need to include not only two or more human parts, but also the contacting parts between the two individuals, resulting in a comparatively complex structure. Secondly, due to the fact that more factors (such as contact parts, contact area, contact method, human body posture and contact pressures) will affect its characteristics, more parameters need to be considered in its mathematical modeling, while some of the parameters are difficult to define. Due to the reasons mentioned above, only the modeling of the IBC via a single body channel has been reported [16-22], few works refer to the modeling of the IBC via handshake channels, and its characteristics remain undisclosed so far.

In this paper, we investigate the modeling of galvanic coupling intra-body communication via handshake channels using the transfer function method. Firstly, we propose a circuit model of the galvanic coupling IBC via handshake channel [23]. Secondly, the modeling process of the contacting parts between two individuals is discussed in detail. Furthermore, the corresponding mathematical model of the whole IBC system is developed. Finally, the validity of the developed model is verified by measurements. The characteristics of the galvanic coupling IBC via handshake channel are compared and discussed with that of the IBC via a single body channel. Our results indicate that the proposed method will lay a foundation for the theoretical analysis and application of the IBC via handshake channel.

\section{Circuit Model}

\subsection{The Whole Circuit Model}

In the galvanic coupling intra-body communication via handshake channel [20], as shown in Figure 1, when two individuals (subject A and B) shake hands; the IBC transmitter attached to subject A detects a triggering signal, prompting it to output digital data through a pair of differential electrodes. The data signal transmits through subject $\mathrm{A}$, the contacting part of the two hands, and subject $\mathrm{B}$. Finally, it is detected by the IBC receiver attached to subject $B$. Therefore, the galvanic coupling intra-body communication between subject $\mathrm{A}$ and $\mathrm{B}$ can be achieved by shaking hands.

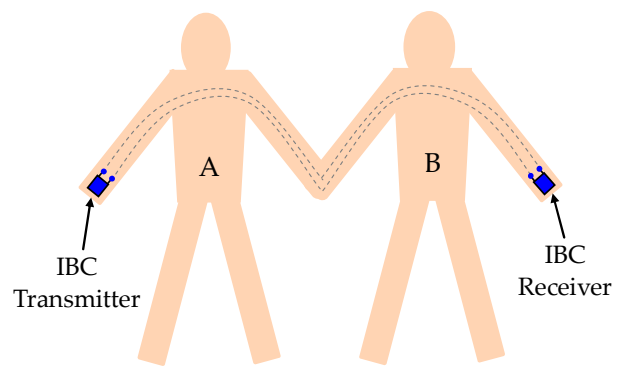

Figure 1. The galvanic coupling intra-body communication between subject $\mathrm{A}$ and $\mathrm{B}$ via handshake channel.

The circuit model of the galvanic coupling IBC via handshake channel is developed by a four-terminal circuit model with discrete complex impedances [10], as shown in Figure 2, which includes the IBC transmitting terminal model, the model of the handshake channel, and the IBC receiving terminal model.

In the IBC transmitting terminal model, as shown in Figure 2, $V_{i}$ represents a voltage source, $R_{o}$ is the internal resistance of the IBC transmitter, and $Z_{\mathcal{C}}$ represents the coupling impedances between the transmitting electrodes and the skin of subject $\mathrm{A}$. 


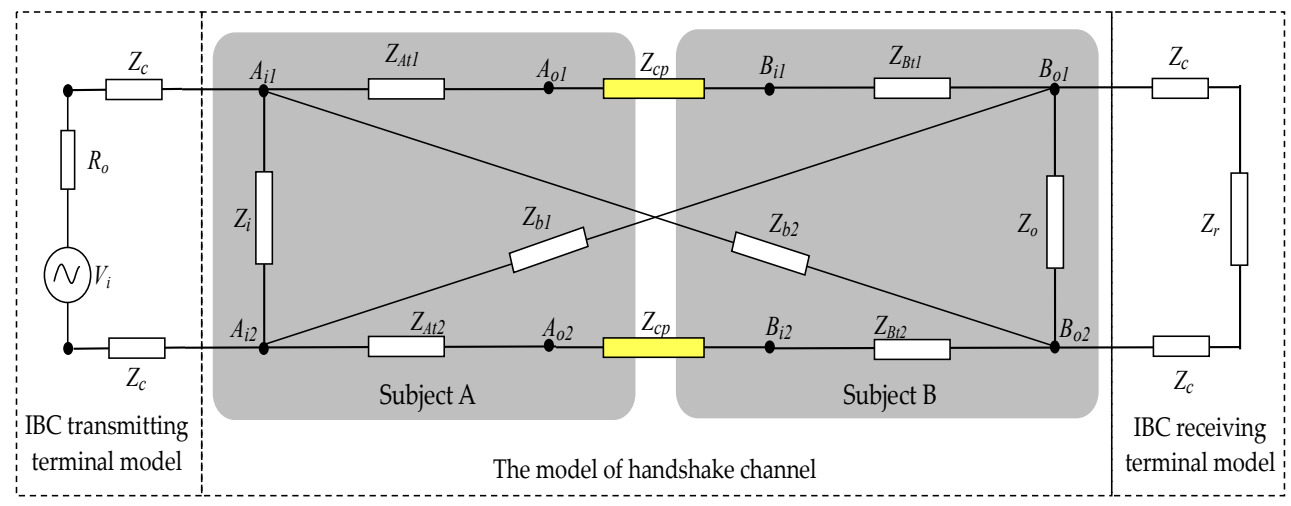

Figure 2. The circuit model of the galvanic coupling intra-body communication via handshake channel.

The model of the handshake channel includes the circuit models of subject $\mathrm{A}$, the contacting parts corresponding to the shaking hands, and subject $B$, respectively. In the circuit models of subject $A$ and $\mathrm{B}, Z_{i}$ and $Z_{o}$ are the input impedance and output impedance of the whole channel, respectively. $Z_{b 1}$ and $Z_{b 2}$ are the cross impedances of the whole channel. On the other hand, $Z_{A t 1}, Z_{A t 2}$ represent the transverse impedances of subject $A$, while the transverse impedances of subject $B$ are represented as $Z_{B t 1}$ and $Z_{B t 2}$. Meanwhile, $Z_{c p}$ represents the contact impedances corresponding to the contacting parts caused by shaking hands, which are very important for the whole circuit model. In the model of the handshake channel, the body impedances except for contact impedances are approximately equivalent to parallel circuits of resistance and capacitance, where parameters are up to Cole-Cole equation [24].

In the IBC receiving terminal model, $Z_{c}$ represents the coupling impedances between the receiving electrodes and the skin of subject $\mathrm{B}$, and $Z_{r}$ represents the input impedance of the receiver in the IBC receiving terminal model $[23,25]$.

\subsection{The Contacting Parts Corresponding to Shaking Hands}

As shown in Figure 3a, the hand of the human body can be divided into palm and fingers. Generally, in the case of shaking hands, the palms of two hands are in close contact with one another, and the palm area is the main area of contact between the two hands. Even if the fingers of one hand also make contact with the back of the other hand, the contact area is comparatively small, and the contact is slight, which can be ignored. As shown in Figure 3b, the contact area has been simplified as two palms in contact with each other for the purposes of our modeling. As shown in Figure 3c, the contacting parts were abstracted as two multi-layer cuboids in contact with each other and which have approximately the same dimensions as palms A and B. Meanwhile, we assumed the cuboids consist of the layers of skin, fat, muscle, cortical bone, and bone marrow [26,27], and that their impedances could be calculated according to their conductivities and relative permittivity [24], as well as the geometric parameters of the palm model, in which the tissue of palm has the same percentage as that of arm.

Based on the geometric modeling described above, the circuit model of $Z_{c p}$ can be derived. As shown in Figure 4, the circuit model of $Z_{c p}$ is abstracted as an impedance network, which consists of a number of unit transverse impedances $\left(\Delta Z_{H}\right)$ and unit longitudinal impedances $\left(\Delta Z_{V}\right)$. Of these, $\Delta Z_{A H}$ and $\Delta Z_{B H}$ represent the transverse impedance per unit length in the palm A model and the palm $B$ model, respectively, while $\Delta Z_{V}$ represents the longitudinal impedances per unit length of the whole model of palm A and palm B. Additionally, there is a containment relationship between the circuit model of Figure 2 and that of Figure 4 . The nodes of $A_{0}$ and $B_{i}$ in Figure 4 correspond to $A_{01} / A_{02}$ and $B_{01} / B_{02}$ in Figure 2, respectively. 


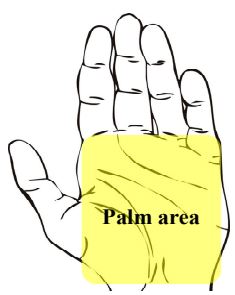

(a)

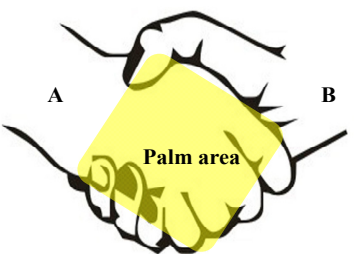

(b)

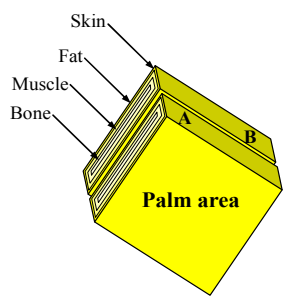

(c)

Figure 3. Modeling of contacting parts corresponding to shaking hands, (a) human hand, (b) handshake gesture, and (c) the modeled palm corresponding to handshake.

$A_{o}$ is the signal input/output point between the subject A model and the palm A model, while $B_{i}$ is the signal input/output point between the palm A model and the subject B model.

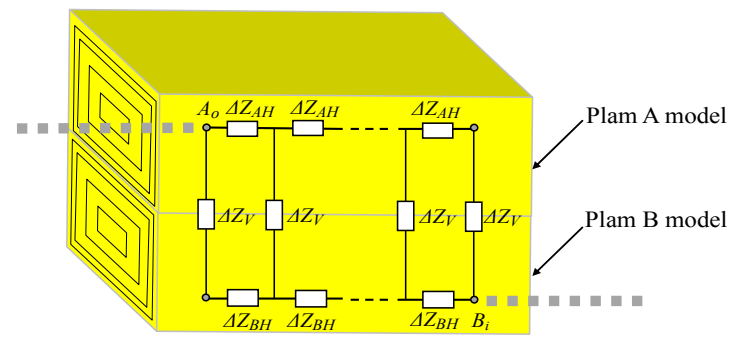

Figure 4. The circuit model of the contacting parts corresponding to shaking hands.

\subsubsection{Transverse Impedance $Z_{H}$}

As shown in Figure 5a, the geometry of the palms is modeled by a cuboid with five layers, including skin, fat, muscle, cortical bone and bone marrow. It is assumed that $L$ and $M$ represent the length and width of the cuboid, respectively, while $\Delta Z_{H}$ represents the unit transverse impedance with the unit length of $\Delta L$, as shown in Figure $5 \mathrm{~b}$. Due to the parallel form of the five-layer tissue shown in Figure $5 b$, the unit transverse impedance $\left(\Delta Z_{H}\right)$ can be expressed as the parallel connection of the impedances corresponding to the five layers, which include the impedances of the layers of the skin $\left(\Delta Z_{s}\right)$, fat $\left(\Delta Z_{f}\right)$, muscle $\left(\Delta Z_{m}\right)$, cortical bone $\left(\Delta Z_{c b}\right)$ and bone marrow $\left(\Delta Z_{b}\right)$, respectively. Finally, the unit transverse impedance $\left(\Delta Z_{H}\right)$ can be determined according to the following equation, which is deduced from the circuit model shown in Figure $5 \mathrm{c}$.

$$
\Delta Z_{A H}=\frac{1}{\sum_{l=1}^{5} \frac{1}{\Delta Z_{l}}}=\frac{1}{\sum_{l=1}^{5}\left(\frac{1}{R_{l}}+j \omega C_{l}\right)}=\frac{\Delta L}{\sum_{l=1}^{5} \sigma_{l f} S_{l}+j \omega \varepsilon_{0} \sum_{l=1}^{5} \varepsilon_{r l f} S_{l}}
$$

where $S_{l}$ is the cross-sectional area of the $l$ th layer. $\sigma_{l f}$ and $\varepsilon_{r l f}$ are the conductivity and relative permittivity corresponding to the different layers and signal frequencies, respectively.

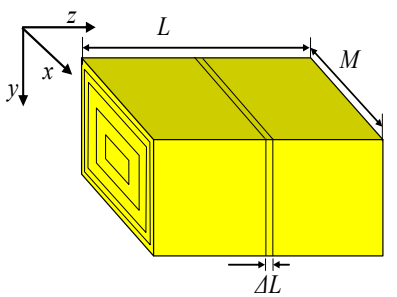

(a)

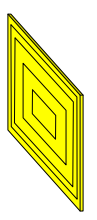

(b)

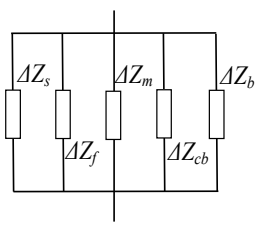

(c)

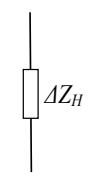

(d)

Figure 5. Determination of $Z_{H}$, (a) the modeled plam, (b) the modeled palm with unit length, (c) the unit impedances corresponding to five-layer tissue, and (d) the unit impedance $\Delta Z_{H}$. 


\subsubsection{Longitudinal Impedance $Z_{V}$}

As shown in Figure 6a, the contacting parts corresponding to the handshake are modeled by two cuboids that are in contact with each other. Meanwhile, $\Delta Z_{V}$ represents the unit longitudinal impedance, with a unit length of $\Delta L$, as shown in Figure $6 \mathrm{~b}$. On the other hand, the models of palm $\mathrm{A}$ and the five-layer tissue can be regarded as a series connection of the five tissue layers along the $y$-axis. Therefore, both the unit transverse impedance of palm $A$ and that of palm $B$ can be expressed as a series connection of the five tissue layers. As a result, both $\Delta Z_{V A}$ and $\Delta Z_{V A B}$ are equal to the sum of $\Delta Z_{s}, \Delta Z_{f}, \Delta Z_{m}, \Delta Z_{c b}$ and $\Delta Z_{b}$, as shown in Figure 6c. Finally, $\Delta Z_{V A}$ and $\Delta Z_{V B}$ can be determined according to Equation (2), and the unit longitudinal impedance $\left(\Delta Z_{V}\right)$ of the model shown in Figure $6 \mathrm{~d}$ is equal to the sum of $\Delta Z_{V A}$ and $\Delta Z_{V B}$.

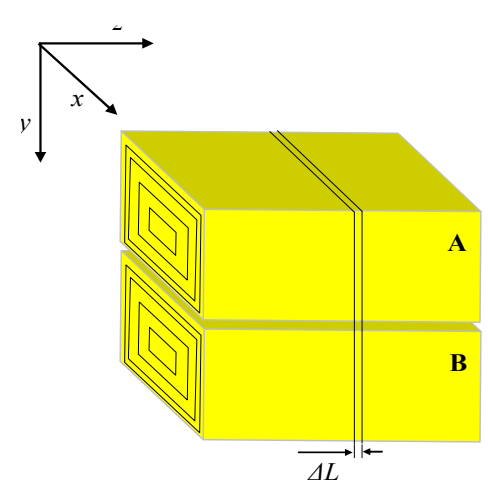

(a)

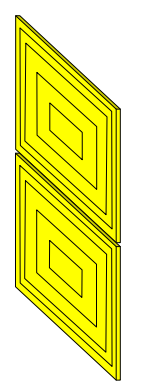

(b)

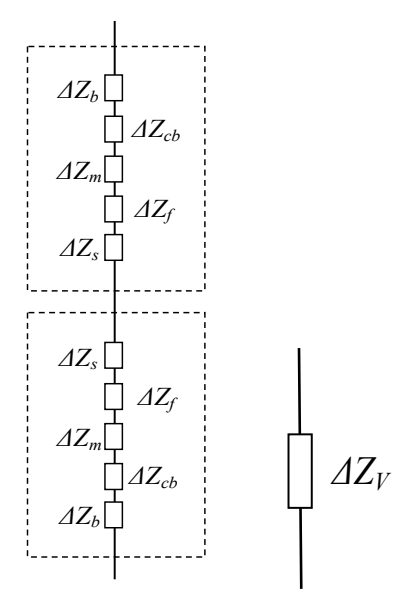

(c) (d)

Figure 6. Determination of $Z_{V}$; (a) the modeled plams corresponding to handshake, (b) the modeled palm with unit length, (c) the unit impedances corresponding to five-layer tissue, and (d) the unit impedance $\Delta Z_{V}$.

$$
\Delta Z_{V A}=\sum_{l=1}^{5} \frac{1}{\Delta Z_{l}}=\sum_{l=1}^{5}\left(\frac{1}{R_{l}}+j \omega C_{l}\right)=\frac{\sum_{l=1}^{5} \sigma_{l f} S_{l}+j \omega \varepsilon_{0} \sum_{l=1}^{5} \varepsilon_{r l f} S_{l}}{\Delta L}
$$

\section{Mathematical Model}

\subsection{The Transfer Function}

Based on the proposed circuit model, a mathematical model of a galvanic coupling IBC can be obtained. The equivalent circuit of the proposed circuit model can be developed using the method of equivalent transformation, as shown in Figure 7.

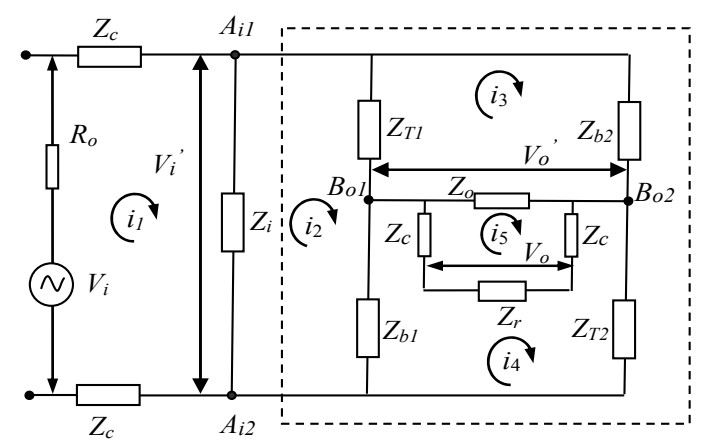

Figure 7. The equivalent circuit of the galvanic coupling IBC via handshake channel. 
According to Figure 2, the series impedance $Z_{t 1}, Z_{c p}$, and $Z_{t 1^{\prime}}$ can be equivalent to $Z_{T 1}$ shown in Figure 7, which is the sum of $Z_{t 1}, Z_{c p}$, and $Z_{t 1^{\prime}}$; while the series impedance $Z_{t 1}, Z_{c p}$ and $Z_{t 1^{\prime}}$ can be equivalent to $Z_{T 1}$ shown in Figure 7 . Therefore, the following equation can be achieved.

$$
\left\{\begin{array}{l}
Z_{T 1}=Z_{A t 1}+Z_{c p}+Z_{B t 1} \\
Z_{T 2}=Z_{A t 2}+Z_{c p}+Z_{B t 2}
\end{array}\right.
$$

The transfer function can be expressed by five linear equations, which can be calculated by a matrix form, as shown as Equation (4).

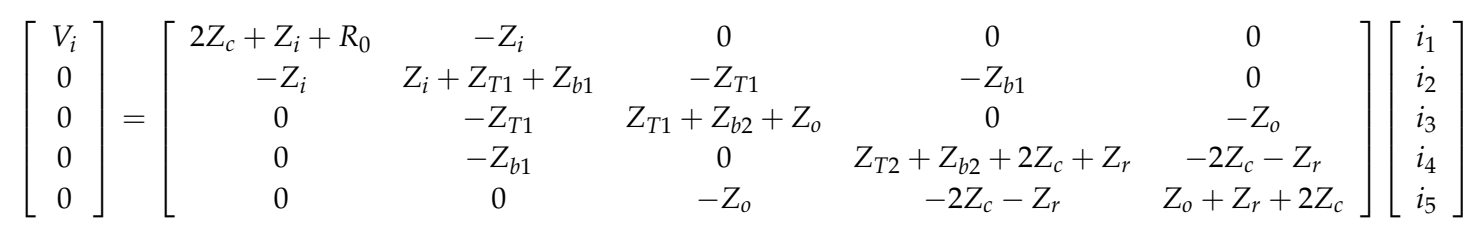

The matrix multiplication form corresponding to Equation (4) is

$$
\mathbf{V}=\mathbf{Z I}
$$

where $\mathbf{V}$ is a column vector, in which the elements represent independent voltage source in each mesh, $\mathbf{Z}$ is a $5 \times 5$ impedance matrix, and $\mathbf{I}$ is a column vector holding mesh currents. Thus, the values of $\mathbf{I}$ can be obtained by multiplying the voltage vector by the inverse matrix of $\mathbf{Z}$, and

$$
\mathbf{I}=\mathbf{Z}^{-1} \mathbf{V}
$$

Therefore, the value of output voltage $V_{o}$ can be calculated as

$$
V_{o}=\left(i_{4}-i_{5}\right) Z_{r}
$$

On the basis of Equation (7), the transfer function $H$ can be obtained,

$$
H=\frac{\left|V_{o}\right|}{V_{i}}
$$

Therefore, the whole transfer function can be expressed as Equation (9),

$$
\left\{\begin{array}{l}
H=\frac{H_{h} Z_{r} Z_{i} Z_{h}}{\left(Z_{r}+2 Z_{c}\right)\left[\left(Z_{i} Z_{h}+\left(2 Z_{C}+R_{0}\right)\left(Z_{i}+Z_{h}\right)\right)\right]} \\
Z_{h}=\frac{Z_{T 1}\left(Z_{T 2} Z_{o}^{\prime}+Z_{b 2} Z_{o}^{\prime}\right)}{\left(Z_{T 1}+Z_{b 2}+Z_{o}^{\prime}-k Z_{o}^{\prime}\right)\left(Z_{o}^{\prime}-Z_{T 2} H_{h}\right)} \\
H_{h}=\frac{(k-1) Z_{o}}{Z_{b 2}+k Z_{T 2}} \\
k=\frac{Z_{b 1} Z_{T 1}+Z_{b 1} Z_{o}^{\prime}+Z_{b 1} Z_{b 2}+Z_{o}^{\prime} Z_{T 1}}{Z_{b 1} Z_{T 1}+Z_{b 1} Z_{o}^{\prime}+Z_{T 2} Z_{T 1}+Z_{o}^{\prime} Z_{T 1}} \\
Z_{T 1}=Z_{A t 1}+Z_{B t 1}+Z_{c p} \\
Z_{T 2}=Z_{A t 2}+Z_{B t 2}+Z_{c p} \\
Z_{o}^{\prime}=Z_{o} / /\left(2 Z_{c}+Z_{r}\right)
\end{array}\right.
$$

Equation (9) shows the whole transfer function of galvanic coupling intra-body communication via handshake channel, which includes the transmitter, subject $\mathrm{A}$, the contacting parts corresponding to shaking hands, subject $B$, and the receiver, and can simulate the whole signal transmission for this kind of IBC. In Equation (9), $H$ represents the whole transfer function; $Z_{h}$ represents the total impedance of dotted portion shown in Figure $7 ; H_{h}$ represents the transfer function of the circuit between $V_{i}{ }^{\prime}$ and $V_{o}{ }^{\prime} ; k$ is the ratio of $i_{4}$ to $i_{3} ; Z_{T 1}$ is an equivalent series impedance formed by $Z_{t 1}, Z_{c p}, Z_{t 1}{ }^{\prime} ; Z_{T 2}$ is 
an equivalent series impedance formed by $Z_{t 2}, Z_{c p}, Z_{t 2}{ }^{\prime}$; and $Z_{o}{ }^{\prime}$ is the impedance corresponding to the $V_{i}{ }^{\prime}$ between nodes $B_{01}$ and $B_{02}$.

Finally, the attenuation of the signal transmission in the galvanic coupling IBC via handshake channel can be determined by

$$
G=20 \log _{10} H+K
$$

where $K$ is the correction factor used for correcting the inherent error between the measurements and the simulations. Generally, $K$ is influenced by the modeling method, the parameter determination method and the measurement precision [23]. A detailed discussion of $K$ is provided in Section 4.2.

\subsection{Determination of $Z_{c p}$}

To determine the value of $Z_{c p}$, the circuit model shown in Figure 4 should be defined. Figure 8a shows the determination method of $Z_{c p}$, and Figure $8 \mathrm{~b}$ is the equivalent circuit of Figure 8a. As illustrated in Figure 8, if voltage is applied at the nodes of $A_{1}, B_{1}$ and $A_{0}$, the corresponding currents of $i_{1}, i_{2}$, and $i_{1}+i_{2}$ can be generated. According to the circuit shown in Figure $8 \mathrm{a}$, the following equation can be obtained.

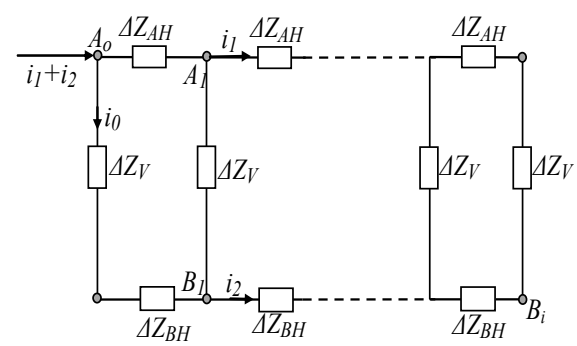

(a)

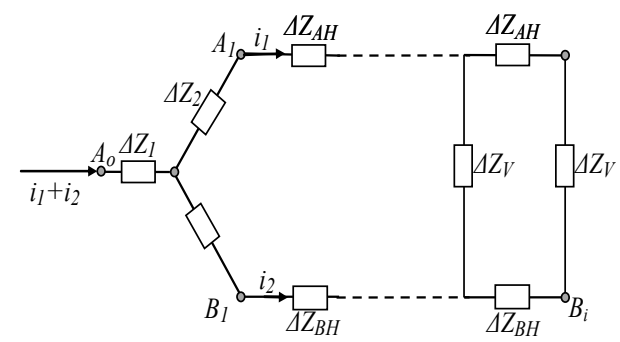

(b)

Figure 8. The determination of $Z_{c p}$. (a) is the analysis of the circuit model shown in Figure 4; (b) is the equivalent circuit of (a).

$$
\left(\Delta Z_{V}+\Delta Z_{B H}\right) i_{0}=\Delta Z_{A H}\left(i_{1}+i_{2}-i_{0}\right)+\Delta Z_{V}\left(i_{2}-i_{0}\right)
$$

As a result, the current of $i_{0}$ can be expressed as

$$
i_{0}=\frac{\Delta Z_{A H} i_{1}+\left(\Delta Z_{A H}+\Delta Z_{V}\right) i_{2}}{\Delta Z_{A H}+\Delta Z_{B H}+2 \Delta Z_{V}}
$$

On the other hand, according to the Kirchhoff's circuit laws, the following equation can be obtained.

$$
\left\{\begin{array}{l}
u_{A_{o} B_{1}}=\left(\Delta Z_{V}+\Delta Z_{B H}\right) i_{0} \\
u_{A_{0} A_{1}}=\Delta Z_{A H}\left(i_{1}+i_{2}-i_{0}\right)
\end{array}\right.
$$

Substitute $i_{0}$ into Equation (13), leading to the following equation:

$$
\left\{\begin{array}{l}
u_{A_{0} B_{1}}=\frac{\Delta Z_{A H}\left(\Delta Z_{V}+\Delta Z_{B H}\right) i_{1}+\left(\Delta Z_{V}+\Delta Z_{B H}\right)\left(\Delta Z_{A H}+\Delta Z_{V}\right) i_{2}}{\Delta Z_{A H}+\Delta Z_{B H}+2 \Delta Z_{V}} \\
u_{A_{0} A_{1}}=\frac{\Delta Z_{A H}\left(\Delta Z_{B H}+2 \Delta Z_{V} i_{1}+\Delta Z_{A H}\left(\Delta Z_{B H}+\Delta Z_{V}\right) i_{2}\right.}{\Delta Z_{A H}+\Delta Z_{B H}+2 \Delta Z_{V}}
\end{array}\right.
$$

Meanwhile, according to the circuit shown in the Figure $8 b$, the following equations can be set up,

$$
\left\{\begin{array}{l}
u_{A_{0} B_{1}}=\Delta Z_{1} i_{1}+\left(\Delta Z_{1}+\Delta Z_{3}\right) i_{2} \\
u_{A_{0} A_{1}}=\left(\Delta Z_{1}+\Delta Z_{2}\right) i_{1}++\Delta Z_{1} i_{2}
\end{array}\right.
$$

According to Equations (14) and (15), the following equation can be obtained. 


$$
\left\{\begin{aligned}
\Delta Z_{1} & =\frac{\Delta Z_{A H}\left(\Delta Z_{V}+\Delta Z_{B H}\right)}{\Delta Z_{A H}+2 \Delta Z_{V}+\Delta Z_{B H}} \\
\Delta Z_{2} & =\frac{\Delta Z_{A H} \Delta Z_{V}}{\Delta Z_{A H}+2 \Delta Z_{V}+\Delta Z_{B H}} \\
\Delta Z_{3} & =\frac{\Delta Z_{V}\left(\Delta Z_{V}+\Delta Z_{B H}\right)}{\Delta Z_{A H}+2 \Delta Z_{V}+\Delta Z_{B H}}
\end{aligned}\right.
$$

Finally, the value of $Z_{c p}$, which represents the contact impedance of the contacting parts corresponding to the shaking hands, can be determined by loop processes.

\section{Measurements and Analysis}

\subsection{Measurement Setup}

An in vivo measurement setup was built to verify the feasibility of the developed mathematical model. Meanwhile, the amplitude-frequency characteristics of the galvanic coupling IBC via handshake channel were carried out.

In our experiments, two males were chosen as the subjects, whose geometry parameters are shown in Table 1, the definitions of the geometry parameters are shown in Figure 9a. The measurement setup was composed of a signal generator (Tektronix AFG3101, $R_{0}=50 \Omega$ ), a digital oscilloscope (Tektronix MSO3012, $Z_{r}=1 \mathrm{M} \Omega$ ), the galvanic coupling electrodes and two high-power lithium power batteries, as shown in Figure 9b. In our measurements, the galvanic coupling electrodes included two pairs of circular copper electrodes with the radius of $10 \mathrm{~mm}$.

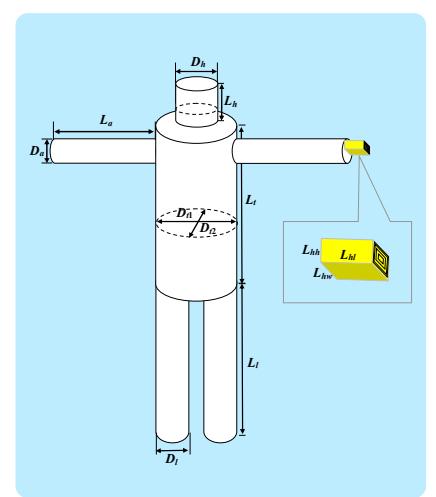

(a)

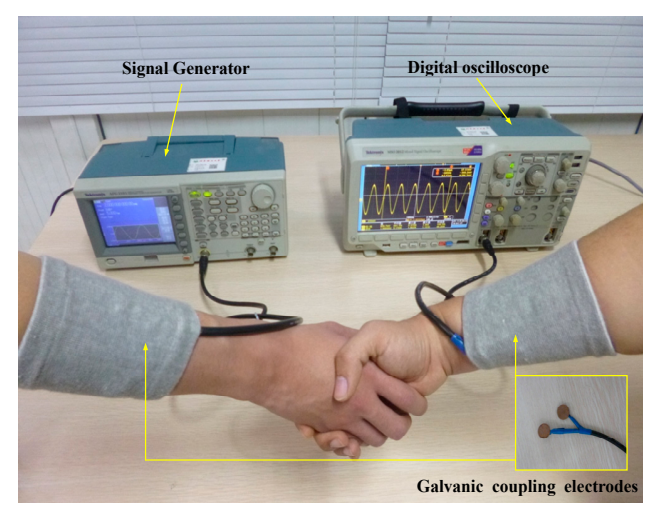

(b)

Figure 9. Parameter definitions and measurement setup. (a) Definitions of the geometry parameters; (b) The measurement setup.

Table 1. Parameters of the subjects.

\begin{tabular}{cccccccccccc}
\hline \multirow{2}{*}{ Subject } & \multirow{2}{*}{ Age } & \multirow{2}{*}{ BMI } & \multirow{2}{*}{ Weight//kg } & Head/cm & Arm/cm & \multicolumn{3}{c}{ Hand/cm } & \multicolumn{2}{c}{ Leg/cm } & \multicolumn{2}{c}{ Torso/cm } \\
\cline { 3 - 12 } & & & & $\boldsymbol{D}_{\boldsymbol{h}}$ & $\boldsymbol{D}_{\boldsymbol{a}}$ & $\boldsymbol{L}_{\boldsymbol{h l}}$ & $\boldsymbol{L}_{\boldsymbol{h} \boldsymbol{w}}$ & $\boldsymbol{L}_{\boldsymbol{h} \boldsymbol{h}}$ & $\boldsymbol{D}_{\boldsymbol{l}}$ & $\boldsymbol{D}_{\boldsymbol{t} \mathbf{1}}$ & $\boldsymbol{D}_{\boldsymbol{t} \mathbf{2}}$ \\
\hline Subject A & 24 & 38.67 & 71 & 18 & 10.0 & 9.2 & 8.1 & 2.0 & 29 & 30 & 20 \\
Subject B & 23 & 20.67 & 67 & 18 & 10.2 & 8.9 & 8.0 & 2.0 & 27 & 29 & 20 \\
\hline
\end{tabular}

Considering the geometry of the arm, both the transmitter electrode and the receiver electrode were attached to the arms at a distance of $6 \mathrm{~cm}$ [27]. Moreover, 10 in vivo measurements were conducted within 10 days to eliminate the influence of time, with the average attenuations being considered to be the measured values in our experiment. In addition, the signal generator output sinusoidal signals had an amplitude of $5 \mathrm{~V}$ (peak-to-peak value), which is below the safety thresholds and avoids resulting in adverse heating effects [19]. The frequency range of $100 \mathrm{kHz}-6 \mathrm{MHz}$ was selected, for which the lower boundary is well above the spectrum of biological signals of the human body. Meanwhile, according to [18], the lumped circuit model is generally valid only when $L c<\lambda$, 
where $L c$ is the circuit's characteristic length, and $\lambda$ is the circuit's operating wavelength. For IBC via handshake channel, due to the fact that two subjects are involved in the signal transmission channel, the dimensions (the circuit's characteristic length) of the IBC via handshake channel is about double that of the IBC via single body channel, which indicates that the operated frequency should be cut in half. Therefore, the upper limit frequency should be set at approximate half of the upper limit frequency $(10 \mathrm{MHz})$ of the IBC via single body channel. As a result, the signal electromagnetic field transmitting within two human bodies can be regarded as a near-field region, which can be modeled using a static circuit model. Finally, the upper limit frequency was set at $6 \mathrm{MHz}$ in our experiments. Additionally, the influence corresponding to the operated frequency between the measurement instruments and the cables was ignored [28-30].

\subsection{Signal Transmission of the Different Distances}

The in vivo measurements along the arms and hands of the two subjects who were shaking hands were carried out. As shown in Figure 10, the transmitting electrodes were placed on the positions of $A_{1}, A_{2}$ and $A_{3}$ of subject $\mathrm{A}$, while the receiving electrodes were placed on $B_{1}, B_{2}$, and $B_{3}$ of subject $\mathrm{B}$, resulting in signal transmission distances of $30 \mathrm{~cm}\left(A_{1}-B_{1}\right), 40 \mathrm{~cm}\left(A_{2}-B_{2}\right)$ and $50 \mathrm{~cm}\left(A_{3}-B_{3}\right)$, respectively. Additionally, according to our measurement experiments, the tiny gap between the two hands had a small influence on the signal attenuation of the IBC via handshake channel. In our subsequent experiments, in order to unify the experimental conditions, the two hands of the two subjects contacted tightly in the processes of shaking hands, and the influence of the tiny gap between the two hands was ignored.

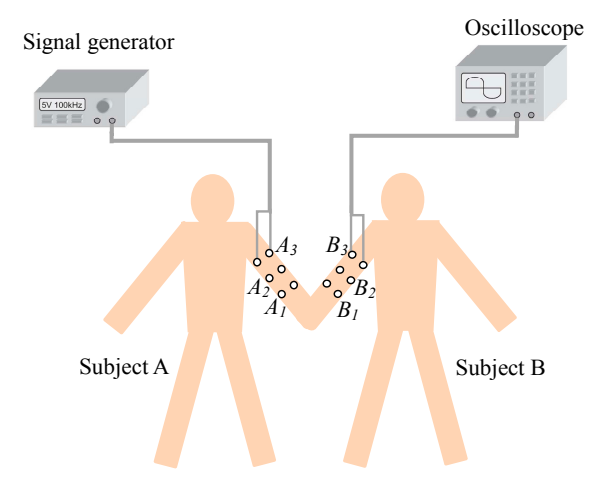

Figure 10. The in vivo measurements of the different signal transmission distances.

Meanwhile, on the basis of the signal transmission distances, electrode size, and geometry parameters of the subjects, the corresponding simulations were carried out using the developed mathematical model. Table 2 shows some of the parameters used in the simulation, which includes $Z_{c}$, $Z_{A t 1} / Z_{A t 2}, Z_{B t 1} / Z_{B t 2}, Z_{i}, Z_{0}, Z_{c p}, R_{0}$ and $Z_{r}$, where $L$ represents the distance between the electrodes of TX and RX.

Table 2. Some parameters used in the simulations.

\begin{tabular}{|c|c|c|c|c|c|c|c|c|}
\hline Frequency & $Z_{c}$ & $Z_{A t 1} / Z_{A t 2}$ & $Z_{B t 1} / Z_{B t 2}$ & $Z_{i}$ & $Z_{o}$ & $Z_{c p}$ & $R_{0}$ & $Z_{r}$ \\
\hline \multirow{2}{*}{$0.1 \mathrm{MHz}$} & $206 \Omega$ & $(534.2 \times \mathrm{L}) \Omega$ & $(505.6 \times \mathrm{L}) \Omega$ & $2083.1 \Omega$ & $2088.7 \Omega$ & $824 \Omega$ & \multirow{4}{*}{$50 \Omega$} & \multirow{4}{*}{$1 \mathrm{M} \Omega$} \\
\hline & $495.0 \mathrm{pF}$ & $(0.386 / \mathrm{L}) \mathrm{nF}$ & $(0.406 / \mathrm{L}) \mathrm{nF}$ & $15.10 \mathrm{pF}$ & $14.3 \mathrm{pF}$ & $111 \mu \mathrm{F}$ & & \\
\hline \multirow{2}{*}{$1 \mathrm{MHz}$} & $126 \Omega$ & $(328.1 \times L) \Omega$ & $(312.5 \times \mathrm{L}) \Omega$ & $1437.9 \Omega$ & $1509.8 \Omega$ & $532.5 \Omega$ & & \\
\hline & $200.5 \mathrm{pF}$ & $(0.135 / \mathrm{L}) \mathrm{nF}$ & $(0.141 / \mathrm{L}) \mathrm{nF}$ & $4.69 \mathrm{pF}$ & $4.467 \mathrm{pF}$ & $405 \mu \mathrm{F}$ & & \\
\hline
\end{tabular}

In our investigation, due to the influences of the simulation method, the parameter determinations and the measurement setup's precision, there is an inherent error between measurement and simulation. As shown in Figure 11, an approximate constant error with a standard deviation of $0.41 \mathrm{~dB}$ can be 
found between measurement results and the corresponding simulation results, which is similar to the case of the IBC via single body channel [23]. In the conventional simulation of the IBC via handshake channel, in order to eliminate this inherent error, a correction factor of $K$ is used, which is equal to the average of the errors $(9.36 \mathrm{~dB})$. If higher precision is needed, the $K$ corresponding to the IBC system should be calibrated. In concrete terms, the measurement should first be carried out under the condition that two individuals are shaking hands. Then, the corresponding simulation should be carried out using the proposed method. Finally, the $K$ corresponding to the IBC system can be determined by calculating the differences between the measurement and simulations.

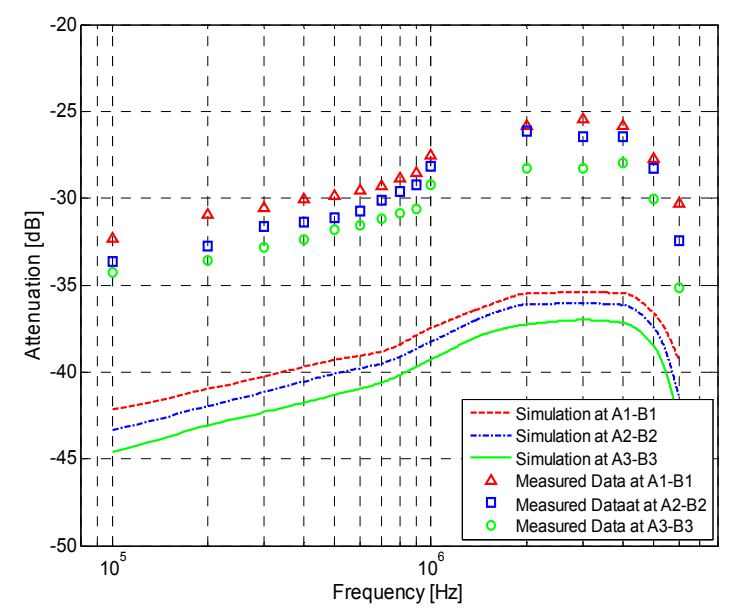

Figure 11. Simulation results and the measurement results of the different signal transmission distances without the correction factor.

The signal attenuations of the measurement and simulation results are shown in Figure 12. The average and standard deviation of the signal at $A_{1}-B_{1}$ over 10 days are shown in Table 3 .

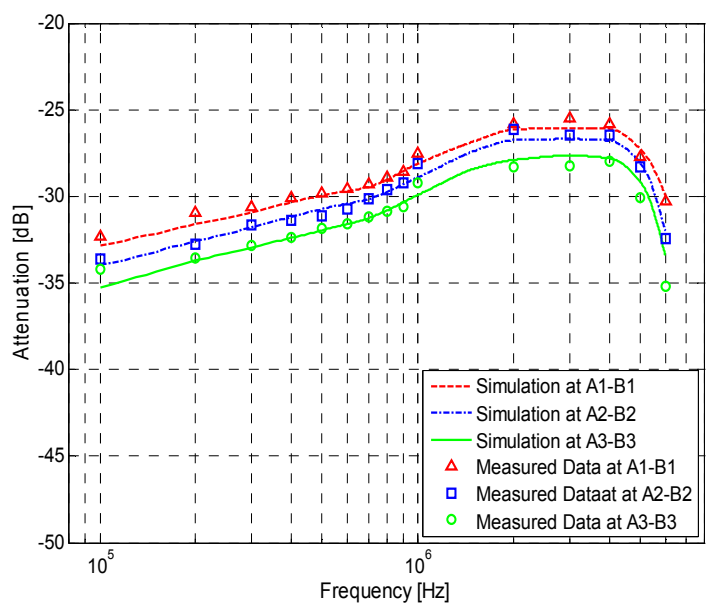

Figure 12. Simulation and measurement results of the signal transmission with different distances.

Table 3. The average and standard deviation of $A_{1}-B_{1}$.

\begin{tabular}{ccccccccc}
\hline Frequency/MHz & $\mathbf{0 . 1}$ & $\mathbf{0 . 2}$ & $\mathbf{0 . 5}$ & $\mathbf{0 . 7}$ & $\mathbf{1}$ & $\mathbf{1 . 5}$ & $\mathbf{3}$ & $\mathbf{5}$ \\
\hline Average $/ \mathrm{dB}$ & -32.32 & -30.93 & -31.11 & -30.11 & -28.13 & -26.12 & -26.44 & -32.46 \\
Standard Deviation $/ \mathrm{dB}$ & 0.75 & 0.87 & 1.07 & 1.34 & 1.37 & 1.63 & 0.88 & 1.40 \\
\hline
\end{tabular}


According to the results shown in Figure 12, some observations can be deduced as follows: (1) The mathematical simulation results basically agree with the corresponding in vivo measurement results. For instance, with a signal frequency of $100 \mathrm{kHz}-2 \mathrm{MHz}$, the average errors between simulations and measurements are only $0.12 \mathrm{~dB}\left(A_{1}-B_{1}\right), 0.26 \mathrm{~dB}\left(A_{2}-B_{2}\right)$ and $0.48 \mathrm{~dB}\left(A_{3}-B_{3}\right)$, respectively; (2) Both the simulation results and the in vivo measurement results decrease as the signal frequency increases from $100 \mathrm{kHz}$ to $2 \mathrm{MHz}$. In a frequency range of $2 \mathrm{MHz}-4 \mathrm{MHz}$, both of them reach their minimum values and remain almost unvaried. As the signal frequency increases from $4 \mathrm{MHz}$ to $6 \mathrm{MHz}$, the simulation and measurement results increase rapidly; (3) As the transmission distance increases, a corresponding increase of the signal attenuations in the measurement and simulation results can also be found. Specifically, an increase of $10 \mathrm{~cm}$ in the signal transmission distance leads to an extra average attenuation of $1.11 \mathrm{~dB}$ in the simulation results, while the corresponding value in the measurement results is $1.20 \mathrm{~dB}$.

\subsection{Signal Transmission within the Different Human Parts}

In the galvanic coupling IBC via handshake channel, IBC devices may be placed on different parts of the body, such as the arm, torso and leg. As a result, signal may be transmitted along different paths within the two human bodies. As shown in Figure 13, simulation and in vivo measurements for different signal transmission paths within two subjects who were shaking hands were carried out, in which the IBC electrodes were placed on the upper arm $\left(A_{1}, B_{1}\right)$, torso $\left(A_{2}, A_{3}, B_{2}, B_{3}\right)$ and thigh $\left(A_{4}, B_{4}\right)$ of the two subjects. The distances of the four paths were $60 \mathrm{~cm}\left(A_{1}-B_{1}\right), 120 \mathrm{~cm}\left(A_{2}-B_{2}\right), 180 \mathrm{~cm}$ $\left(A_{3}-B_{3}\right)$ and $240 \mathrm{~cm}\left(A_{4}-B_{4}\right)$, respectively.

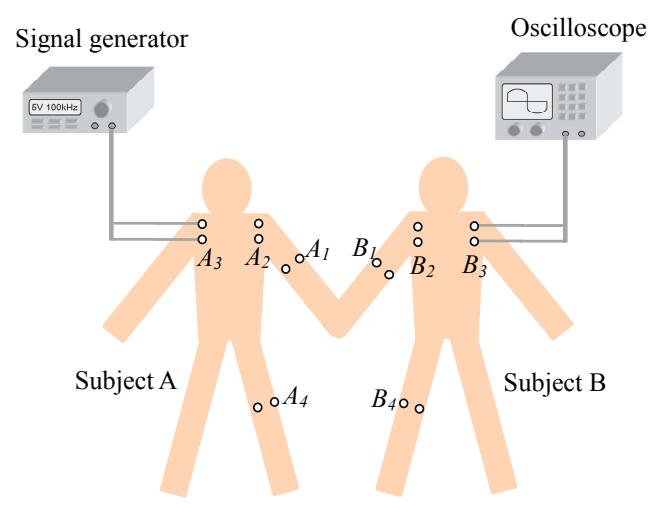

Figure 13. The in vivo measurements of signal transmission between different body parts of the two subjects.

Figure 14 shows the simulation and measurement results of the signal transmission between the different body parts. According to Figure 14, some observations can be deduced as follows: (1) the mathematical simulation results coincide with the measurement results; (2) all of the signal attenuation curves corresponding to the different body parts have similar outlines; (3) both the measurement and that of the simulation increase with the increment of the transmission distance.

In addition, according to Figure 14, signal transmission paths of the different human parts influence the signal attenuations to some extent. For instance, the extra average attenuation of the $A_{2}-B_{2}$ path $(120 \mathrm{~cm})$ increased by $3.48 \mathrm{~dB}$ (measurement) and $3.62 \mathrm{~dB}$ (simulation) compared with that of the $A_{1}-B_{1}(60 \mathrm{~cm})$ path. Meanwhile, compared with that of the $A_{2}-B_{2}(120 \mathrm{~cm})$ path, the extra average attenuation of the $A_{3}-B_{3}$ path $(180 \mathrm{~cm})$ and the $A_{4}-B_{4}$ path $(240 \mathrm{~cm})$ increased by $0.78 \mathrm{~dB}$ (measurement) and $1.49 \mathrm{~dB}$ (measurement), respectively.

It can be found that the above increments are less than that of the IBC based on a single human body $[21,26,27]$, which can be explained by the fact that, over the same distance, the total impedance of the channel including the handshake and arm in the IBC via handshake channel is less than that of 
the arm channel in the IBC based on a single human body. Therefore, the impedance corresponding to the unit distance of the channel including handshake and arm is also less than that of the arm channel, which results in the increase in distance along the arm path having less influence on signal attenuation in the IBC via handshake channel. Further research is needed to clarify this issue in future work.

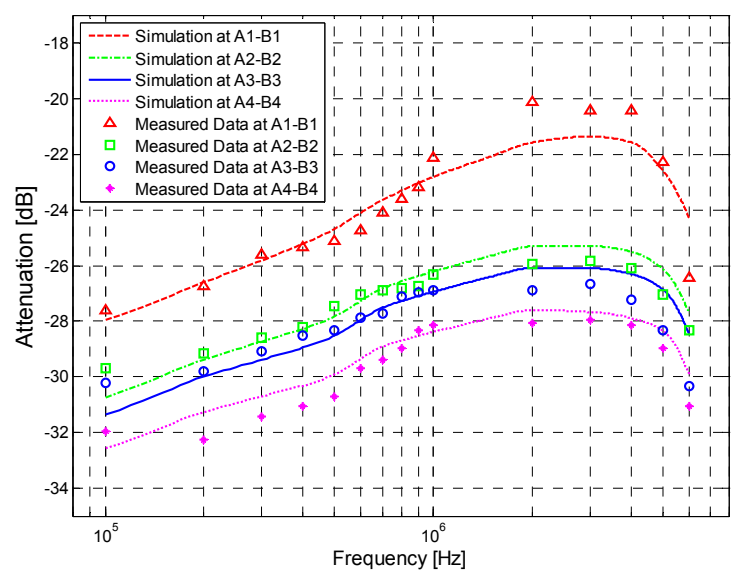

Figure 14. Simulation and measurement results of signal transmission within different body parts.

Moreover, according to [23], the transverse impedance corresponding to unit length of a particular human body part decreases with the increase in cross-sectional area, while the cross-sectional area of the torso is generally two orders bigger than that of arm [31]. Therefore, the transverse impedance of torso is far less than that of the arm under the same conditions, which results in the attenuation differences between path $A_{2}-B_{2}$ and path $A_{3}-B_{3}$ being relatively small, while the attenuation differences between $A_{1}-B_{1}$ path and $A_{2}-B_{2}$ path are relatively big [26].

\subsection{Comparison between Handshake Channel and Single Body}

To compare the characteristics between the IBC via handshake channel and the IBC via single body channel, in vivo measurements were carried out. As shown in Figure 15a, signal was transmitted along the arms of the two subjects, where the distances were $30 \mathrm{~cm}\left(A_{1}-B_{1}\right)$ and $40 \mathrm{~cm}\left(A_{2}-B_{2}\right)$, respectively. Figure $15 \mathrm{~b}$ shows the IBC via single body channel, for which the transmitting electrodes were placed on the position of $A_{1}$, while the receiving electrodes were placed on $A_{2}$ or $A_{3}$, resulting in signal transmission distances of $30 \mathrm{~cm}\left(A_{1}-A_{2}\right)$ and $40 \mathrm{~cm}\left(A_{1}-A_{3}\right)$, respectively. Therefore, the difference between Figure 15a,b is the contact impedance $\left(Z_{c p}\right)$ corresponding to the contacting parts caused by shaking hands.

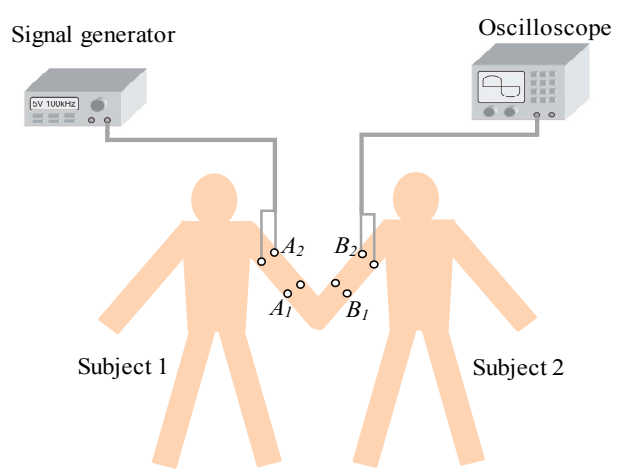

(a)

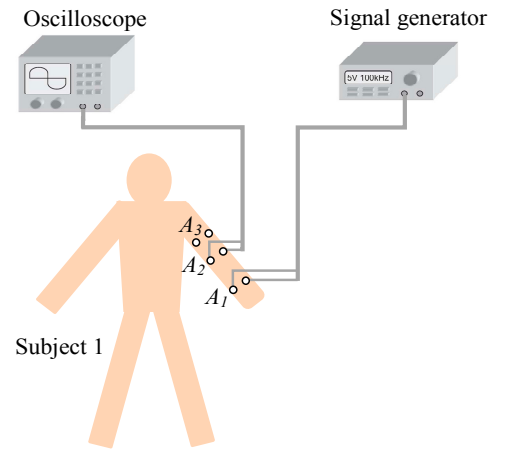

(b)

Figure 15. The in vivo measurements of the signal transmission characteristics. (a) IBC via handshake channel; (b) IBC via single body channel. 
Figure 16 shows the simulation and measurement results of the IBC via handshake channel and the IBC via single body channel. The $K$ of Figure $16 \mathrm{a}$ is $9.36 \mathrm{~dB}$, while the $K$ of Figure $16 \mathrm{~b}$ is $20.28 \mathrm{~dB}$, which is the value of correction factor for the IBC via single body channel [23]. We can find that, due to the influence of $Z_{c p}$, the results of Figure 16a are different from those of Figure 16b, which are described as follows: (1) the signal attenuation of the handshake channel is much bigger than that of the single body channel. According to the simulation results, the maximum and minimum differences between them are $4.80 \mathrm{~dB}(1.05 \mathrm{MHz}, 60 \mathrm{~cm})$ and $1.71 \mathrm{~dB}(220 \mathrm{kHz}, 40 \mathrm{~cm})$, respectively; (2) the peak frequency of the galvanic coupling IBC via handshake channel is within the range of 2-4 MHz, while that of the IBC via single body channel is within the range of $1.5-2.1 \mathrm{MHz}$; (3) compared with the results for single body, the signal attenuations of the handshake channel decrease more slowly in the frequency range of $100 \mathrm{kHz}-2 \mathrm{MHz}$. In concrete terms, the results of $A_{1}-B_{1}$ decrease $6.97 \mathrm{~dB}$ while the corresponding value of $A_{1}-A_{2}$ is $8.44 \mathrm{~dB}$ in this range. Meanwhile, the signal attenuations for the handshake channel increase more rapidly in the signal frequency range of 4-6 MHz. For instance, the results of $A_{1}-B_{1}$ increased by $4.61 \mathrm{~dB}$, while the corresponding value of $A_{1}-A_{2}$ was only $2.16 \mathrm{~dB}$ in this range.

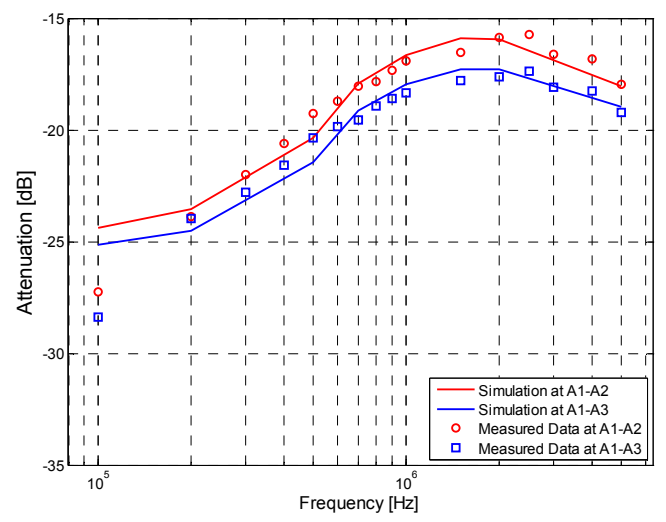

(a)

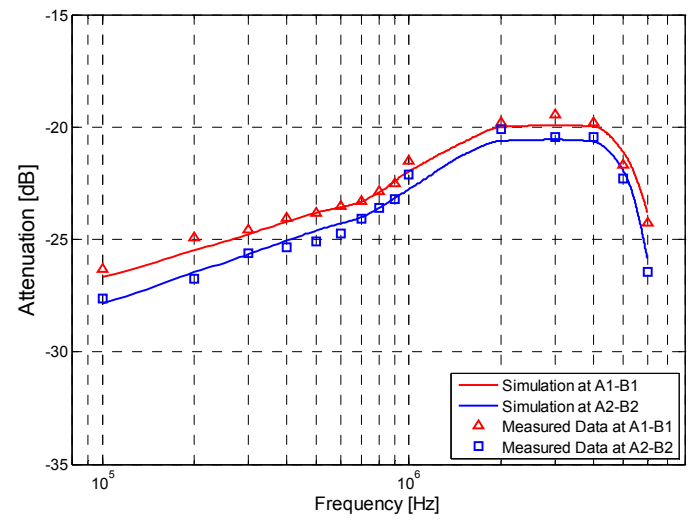

(b)

Figure 16. Simulation and measurement results of the galvanic coupling intra-body communication (a) Results for the galvanic coupling intra-body communication via handshake channel; (b) Results for the IBC via single body channel.

\subsection{Influence of the Distance between the Inter-Electrodes}

In order to investigate the influence of the distance between the inter-electrodes on the galvanic coupling intra-body communication via handshake channel, the corresponding measurements and simulations were carried out. Firstly, the distance between the inter-electrodes of the transmitter, which corresponds to $Z_{i}$, was set at $5 \mathrm{~cm}, 6 \mathrm{~cm}, 7 \mathrm{~cm}, 8 \mathrm{~cm}$ and $9 \mathrm{~cm}$, respectively, while the distance corresponding to $Z_{o}$ at the receiver was fixed at $6 \mathrm{~cm}$, and the signal transmission distance was $30 \mathrm{~cm}$. The results are shown in Figure 17a. Then, the distance corresponding to $Z_{i}$, was fixed at $6 \mathrm{~cm}$, while the distance corresponding to $Z_{o}$ was set at $5 \mathrm{~cm}, 6 \mathrm{~cm}, 7 \mathrm{~cm}, 8 \mathrm{~cm}$ and $9 \mathrm{~cm}$, respectively. The results corresponding to this case are shown in Figure 17b.

According to the results of Figure 17, when the distances between the inter-electrodes increased from $5 \mathrm{~cm}$ to $9 \mathrm{~cm}$, the corresponding decrease of signal attenuations was found, which indicates that the distance between the inter-electrodes does influence the signal attenuation of the galvanic coupling IBC via handshake channel. Meanwhile, the influence of the distance between the inter-electrodes is comparatively small. In concrete terms, according to Figure 17a, an increase of $1 \mathrm{~cm}$ distance between the inter-electrodes led to an extra average attenuation of $0.334 \mathrm{~dB}$ in the simulation results, while the corresponding value of Figure $17 \mathrm{~b}$ was $0.343 \mathrm{~dB}$. 


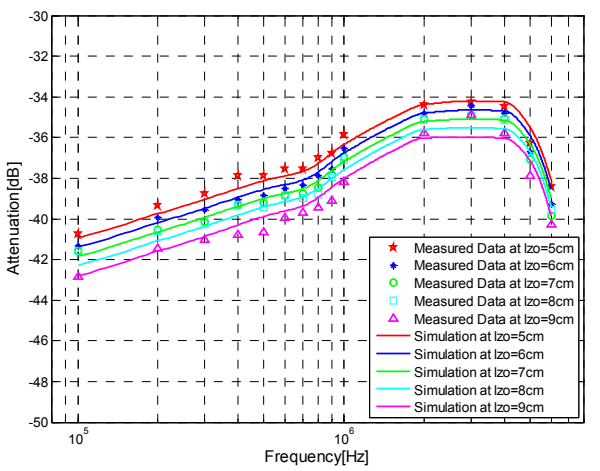

(a)

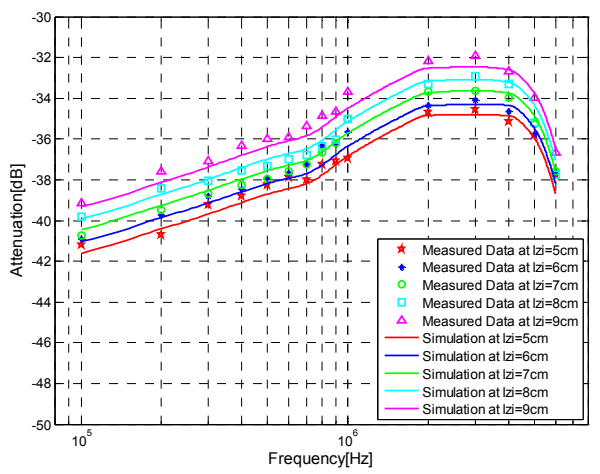

(b)

Figure 17. Simulation results and the measurement results corresponding to $Z_{i}$ and $Z_{o}$. (a) Results corresponding to different inter-electrodes distances of $Z_{0}$, (b) Results corresponding to different inter-electrodes distances of $Z_{i}$.

\section{Conclusions}

In this paper, a method for modeling the galvanic coupling IBC via handshake channel is proposed, while a corresponding mathematical model is developed. Finally, the validity of the developed model is verified by in vivo measurements. Moreover, the characteristics of the galvanic coupling IBC via handshake channel are compared with those of the IBC via single body channel. Our results indicate that the proposed method will offer significant advantages in the theoretical analysis and application of the IBC via handshake channel.

Some conclusions are, therefore, listed as follows: (1) the simulation results based on the proposed mathematical model basically agree with the corresponding in vivo measurement results; (2) the signal attenuations of the galvanic coupling IBC via handshake channel decrease in the frequency range of $100 \mathrm{kHz}-2 \mathrm{MHz}$, reach their minimum values at $2-4 \mathrm{MHz}$, and increase rapidly at $4-6 \mathrm{MHz}$; (3) the signal attenuations of the galvanic coupling IBC via handshake channel are bigger than that of the IBC via single body channel. Meanwhile, the peak frequency of the galvanic coupling IBC via handshake is within $2-4 \mathrm{MHz}$, and that of the IBC via single body channel is within 1.5-2.1 MHz; (4) the distance between the inter-electrodes influences the signal actuation of the galvanic coupling IBC via handshake channel, while the extra average attenuation corresponding to the increase of $1 \mathrm{~cm}$ distance is less than $0.5 \mathrm{~dB}$.

In our future work, the modeling method and the achieved characteristics of the galvanic coupling IBC via handshake channel will be verified by using small battery-operated IBC transmitter and receiver, while the implementation method of the information interaction based on handshake channel will be investigated.

Acknowledgments: The work described in this paper was supported by the National Natural Science Foundation of China (81671787), the Excellent Talent Fund of Beijing, China (3040036521101). The authors would like to thank Jiamiao Yang of Biomedical Engineering at Washington University in St. Louis and Qingyue Song of the High School Attached to Tsinghua University for their help.

Author Contributions: Maoyuan Li, Yong Song and Wansong Li conceived of the whole study, and participated in its design and drafted the complete manuscript. Guangfa Wang, Tianpeng Bu and Yufei Zhao participated in the measurements of all subjects, and Qun Hao gave valuable suggestions and constructive discussions and contributed to manuscript preparation. All authors read and approved the final manuscript.

Conflicts of Interest: The authors declare no conflict of interest.

\section{Abbreviations}

The following abbreviations are used in this manuscript:

IBC intra-body communication

BSN body sensor network 


\section{References}

1. Zimmerman, T.G. Personal Area Networks (PAN): Near-Field Intra-Body Communication; Massachusetts Institute of Technology: Cambridge, MA, USA, 1995.

2. Gravina, R.; Alinia, P.; Ghasemzadeh, H.; Fortino, G. Multi-sensor fusion in body sensor networks: State-of-the-art and research challenges. Inf. Fusion 2017, 35, 68-80. [CrossRef]

3. Fortino, G.; Giannantonio, R.; Gravina, R.; Kuryloski, P.; Jafari, R. Enabling Effective Programming and Flexible Management of Efficient Body Sensor Network Applications. IEEE Trans. Hum. Mach. Syst. 2013, 43, 115-133. [CrossRef]

4. Iyengar, S.; Bonda, F.T.; Gravina, R.; Guerrieri, A.; Fortino, G.; Sangiovanni-Vincentelli, A. A framework for creating healthcare monitoring applications using wireless body sensor networks. In Proceedings of the ICST 3rd International Conference Body Area Networks, Tempe, AZ, USA, 13-17 March 2008.

5. Shinagawa, M.; Fu, M.; Ochiai, K.; Kyuragi, H. A near-field-sensing transceiver for intrabody communication based on the electrooptic effect. IEEE Trans. Instrum. Meas. 2004, 6, 1533-1538. [CrossRef]

6. Cho, N.; Yoo, J.; Song, S.J.; Lee, J.; Jeon, S.; Yoo, H.J. The human body characteristics as a signal transmission medium for intrabody communication. IEEE Trans. Microw. Theory Tech. 2007, 55, 1080-1085. [CrossRef]

7. Fujikawa, M.; Nishigaki, M. A Study of Prevention for Social Engineering Attacks Using Real/Fake Organization's Uniforms: Application of Radio and Intra-Body Communication Technologies. In Proceedings of the Sixth International Conference on Availability, Reliability and Security, Vienna, Austria, 22-26 August 2011; pp. 597-602.

8. Shimamoto, S.; Alsehab, A.M.; Kobayashi, N.; Dovchinbazar, D.; Ruiz, J.A. Future applications of body area communications. In Proceedings of the 6th International Conference on Information, Communications \& Signal Processing, Singapore, 10-13 December 2007; pp. 1-5.

9. Ruiz, J.A.; Shimamoto, S. Novel communication services based on human body and environment interaction: Applications inside trains and applications for handicapped people. In Proceedings of the IEEE Wireless Communications and Networking Conference, Las Vegas, NV, USA, 3-6 April 2006; pp. 2240-2245.

10. Zimmerman, T.G. Personal Area Networks: Near-field intrabody. IBM Syst. J. 1996, 35, 609-617. [CrossRef]

11. Hachisuka, K.; Terauchi, Y.; Kishi, Y.; Sasaki, K.; Hirota, T.; Hosaka, H.; Fujii, K.; Takahashi, M.; Ito, K. Simplified circuit modeling and fabrication of intrabody communication devices. Sens. Actuators A Phys. 2006, 130-131, 322-330. [CrossRef]

12. Hachisuka, K.; Nakata, A.; Takeda, T.; Shiba, K.; Sasaki, K.; Hosaka, H.; Itao, K. Development of wearable intra-body communication devices. Sens. Actuators A Phys. 2003, 105, 109-115. [CrossRef]

13. Ferguson, J.E.; Redish, A.D. Wireless communication with implanted medical devices using the conductive properties of the body. Expert Rev. Med. Devices 2011, 8, 427-433. [CrossRef] [PubMed]

14. Matsushita, N.; Tajima, S.; Ayatsuka, Y.; Rekimoto, J. Wearable key: Device for personalizing nearby environment. Digest of Papers. In Proceedings of the Fourth International Symposium on Wearable Computers, Atlanta, GA, USA, 2000.

15. Chen, X.M.; Mak, P.U.; Pun, S.H.; Gao, Y.M.; Lam, C.T.; Vai, M.I.; Du, M. Study of Channel Characteristics for Galvanic-Type Intra-Body Communication Based on a Transfer Function from a Quasi-Static Field Model. Sensors 2012, 12, 16433-16450. [CrossRef] [PubMed]

16. Chen, X.M.; Pun, S.H.; Zhao, J.F.; Mak, P.U.; Liang, B.D.; Vai, M.I. Effects of human limb gestures on galvanic coupling intra-body communication for advanced healthcare system. Biomed. Eng. Online 2016, 15, 60. [CrossRef] [PubMed]

17. Vai, M.I.; Du, M.; Gao, Y.M.; Wu, Z.M.; Pun, S.H.; Mak, P.U. A Novel Field-Circuit FEM Modeling and Channel Gain Estimation for Galvanic Coupling Real. Sensors 2016, 16, 471.

18. Kibret, B.; Seyedi, M.; Lai, D.T.H.; Faulkner, M. Investigation of galvanic-coupled intrabody communication using the human body circuit model. IEEE J. Biomed. Health Inform. 2014, 18, 1196-1206. [CrossRef] [PubMed]

19. Hachisuka, K.; Terauchi, Y.; Kishi, Y.; Hirota, T.; Sasaki, K.; Hosaka, H.; Ito, K. Simplified circuit modeling and fabrication of intrabody communication devices. In Proceedings of the 13th International Conference on Solid-State Sensors, Actuators and Microsystems, Seoul, Korea, 5-9 June 2005.

20. Wegmueller, M.S. Intra-Body Communication for Biomedical Sensor Networks. Ph.D. Thesis, Swiss Federal Institude of Technology Zurich (ETH), Zurich, Switzerland, 2007. 
21. Pun, S.H.; Gao, Y.M.; Mak, P.; Vai, M.I.; Du, M. Quasi-static modeling of human limb for intra-body communications with experiments. IEEE Trans. Inf. Technol. Biomed. 2011, 15, 870-876. [PubMed]

22. Kagimoto, K.; Anzai, D.; Wang, J. Approaching direction detection of human arm using human body communication technology. In Proceedings of the 2012 Asia-Pacific Symposium on Electromagnetic Compatibility, Singapore, 21-24 May 2012; pp. 461-464.

23. Song, Y.; Hao, Q.; Zhang, K.; Wang, M.; Chu, Y.F.; Kang, B.Z. The simulation method of the galvanic coupling intrabody communication with different signal transmission paths. IEEE Trans. Instrum. Meas. 2011, 60, 1257-1266. [CrossRef]

24. Gabriel, S.; Lau, R.W.; Gabriel, C. The dielectric properties of biological tissues: III. Parametric models for the dielectric spectrum of tissues. Phys. Med. Biol. 1996, 41, 2271-2293. [CrossRef] [PubMed]

25. Callejon, M.A.; Reina-Tosina, J.; Naranjo-Hernandez, D.; Roa, L.M. Galvanic coupling transmission in intrabody communication: A finite element approach. IEEE Trans. Biomed. Eng. 2014, 61, 775-783. [CrossRef] [PubMed]

26. Wegmueller, M.S.; Kuhn, A.; Froehlich, J.; Oberle, M.; Felber, N.; Kuster, N.; Fichtner, W. An attempt to model the human body as a communication channel. IEEE Trans. Biomed. Eng. 2007, 54, 1851-1857. [CrossRef] [PubMed]

27. Wegmueller, M.S.; Oberle, M.; Felber, N.; Kuster, N.; Fichtner, W. Signal Transmission by Galvanic Coupling through the Human Body. IEEE Trans. Instrum. Meas. 2010, 59, 963-969. [CrossRef]

28. Ito, K.; Hotta, Y. Signal Path Loss Simulation of Human Arm for Galvanic Coupling Intra-Body Communication Using Circuit and Finite Element Method Models. In Proceedings of the 2015 IEEE Twelfth International Symposium Auton Decentralized System, Taichung, Taiwan, 25-27 March 2015; pp. 230-235.

29. Callejón, M.A.; Naranjo-Hernández, D.; Reina-Tosina, J.; Roa, L.M. A Comprehensive Study into Intrabody Communication Measurements. IEEE Trans. Instrum. Meas. 2013, 62, 2446-2455. [CrossRef]

30. Gao, Y.-M.; Wu, Z.-M.; Pun, S.-H.; Mak, P.-U.; Vai, M.-I.; Du, M. A Novel Field-Circuit FEM Modeling and Channel Gain Estimation for Galvanic Coupling Real IBC Measurements. Sensors 2016, 16, 471. [CrossRef] [PubMed]

31. Song, Y.; Zhang, K.; Hao, Q.; Hu, L.; Wang, J.; Shang, F. A Finite-Element Simulation of Galvanic Coupling Intra-Body Communication Based on the Whole Human Body. Sensors 2012, 12, 13567-13582. [CrossRef] [PubMed]

(C) 2017 by the authors. Licensee MDPI, Basel, Switzerland. This article is an open access article distributed under the terms and conditions of the Creative Commons Attribution (CC BY) license (http:/ / creativecommons.org/licenses/by/4.0/). 